\title{
Hierarchical Modeling Control for Five-degree-of-freedom Vehicle Model with Four-wheel-independent-controllable Suspension
}

\author{
Long $\mathrm{Wu}^{1,2}$ and Chi-Hsin Yang ${ }^{1 *}$ \\ ${ }^{1}$ School of Mechanical and Electric Engineering, Sanming University, Sanming 36500, Fujian Province, China \\ ${ }^{2}$ Fujian Public Service Platform of mini-EREV Power System, Sanming University, Sanming 36500, \\ Fujian Province, China
}

(Received September 20, 2019; accepted March 18, 2020)

Keywords: four-wheel-independent-controllable suspension (4WICS), hierarchical modeling control method, vibrational sensor, center control, local control

In this study, the configuration of a five-degree-of-freedom vehicle model with four-wheelindependent-controllable suspension (4WICS) is introduced. By taking into account the vertical road and lateral excitations generated from the tire effect, a full-vehicle dynamical model is constructed by space force analysis. The model can be set up using vibrational sensors with the appropriate arrangement for experiments. In this model, on the basis of the kinetic relationships between the locations of the four wheels and the center of the vehicle sprung mass, the ratio relationships of coupling dynamics between one full-vehicle controller and four quarter-vehicle controllers are deduced. Using the introduced dynamical model, we propose the 4WICS control framework on the basis of a hierarchical modeling control method, which consists of a center control layer and four local control layers. The results show that the system responses become rapid and the ride comfort is enhanced. Moreover, owing to the realization of the parallel computation of four local control layers, the vehicle handling properties are improved.

\section{Introduction}

A vehicle suspension system with good performance should not only support the total weight, but also effectively reduce the impact of external excitations and improve adhesion between the wheels and the road. In recent decades, with the development of many new technologies and achievements, active and semiactive suspensions have been applied successively. Several advanced control schemes, such as optimal control, ${ }^{(1)}$ adaptive control, ${ }^{(2-5)}$ fault-tolerant control, ${ }^{(6-9)}$ robust event-triggered control, ${ }^{(10,11)}$ a dynamic programming approach, ${ }^{(12)}$ fuzzy control, ${ }^{(13,14)}$ fuzzy logic control, ${ }^{(15)}$ neuro-fuzzy control, ${ }^{(16-18)}$ adaptive back-stepping control, ${ }^{(19,20)}$ sliding mode control, ${ }^{(21,22)}$ and fractional-order sliding mode control, were proposed. $^{(23)}$

By considering a full-vehicle model, Chen et al. presented an efficient optimal scheme to solve a passive mechanical control problem. ${ }^{(1)}$ However, practical vehicle suspension systems always suffer from component parameter variations. To overcome these issues, many

*Corresponding author: e-mail: 20190207@fjsmu.edu.cn https://doi.org/10.18494/SAM.2020.2685 
adaptive control schemes for tackling unknown nonlinearities in vehicle active suspensions were proposed. ${ }^{(2-5)}$ In addition, in the presence of actuator faults, model uncertainties, and parameter variations, fault-tolerant control strategies were developed on the basis of the sliding mode method, ${ }^{(7)}$ finite-frequency constraints, ${ }^{(8)}$ and output feedback control. ${ }^{(6,9)}$ Recently, a robust event-triggered control system ${ }^{(10,11)}$ has been investigated under parameter uncertainties in the active suspension system of vehicles. In such systems, an event-triggered communicated mechanism is applied to determine which appropriate control input is to be updated according to the suspension system dynamic evolution. The dynamic programming approach ${ }^{(12)}$ with adaptive optimal control is used to achieve performance requirements, such as ride comfort, suspension space limitation, and road holding.

With the desired performance taken into account, the Takagu-Sugeno (TS) fuzzy model was established for uncertain vehicle suspension systems. Then, the parallel-distributed compensation scheme was used for evaluating active suspension controllers. ${ }^{(13,14)}$ The neuro-fuzzy control, ${ }^{(16-18)}$ which combines the fuzzy logic and neural network with the wavelet technique, was proposed to improve the dynamic response based on a nonlinear fullvehicle active suspension system. For active suspension systems with nonlinearity, system uncertainties, and external disturbance, such as unknown road profiles, state-observer-based sliding mode controllers ${ }^{(21-23)}$ were proposed. Moreover, adaptive back-stepping control ${ }^{(19,20)}$ was also developed by introducing a virtual input control signal to stabilize state errors.

In previous works, the vehicle suspension control system was taken into consideration by utilizing two-degree-of-freedom (2-DOF) models (sprung and unsprung mass models) ${ }^{(5,10,11,22,23)}$ or the half-vehicle model. ${ }^{(19)}$ Despite these many achievements, in our present study, we developed a novel 5-DOF vehicle model with a four-wheel-independent-controllable suspension (4WICS) for solving the control problem of a vehicle suspension system. On the basis of 4WICS, a full-vehicle dynamical model was constructed by space force analysis, taking into account the vertical road and lateral excitations generated from the tire effect. Since various smart sensors and microprocessors have emerged for the vehicle suspension system, many signals such as displacements and velocities are available. ${ }^{(10,24)}$ The system parameters of a full-vehicle dynamical model can be analyzed using system identification technologies. ${ }^{(25-28)}$ Thus, the following model of 4WICS that we introduced can be set up using sensors with the appropriate experimental arrangement.

The concept of 4WICS stems from the sprung mass variation of a quarter-vehicle suspension. The determination of the sprung mass still depends on the sophistication and accuracy of the proposed strategies. ${ }^{(25-28)}$ The sprung masses of a full-vehicle suspension and quarter-vehicle suspensions are related and show dynamical coupling relationships. If the coupling ratio relationships between a full-vehicle suspension and quarter-vehicle suspensions are solved, each sprung mass of a quarter-vehicle suspension can be determined under any condition. Therefore, each quarter-vehicle suspension can be treated as an independent controllable unit. In this study, a vehicle suspension system model, 4WICS, which may be simpler than a traditional vehicle suspension model, was constructed. Then, a hierarchical modeling control configuration, which consists of a center control layer and four local control layers, was proposed to achieve a higher vehicle suspension performance. This hierarchical modeling control configuration has the 
advantage that it realizes parallel computations for controlled variables of four quarter-vehicle suspensions.

The rest of this paper is organized as follows. In Sect. 2, the dynamic decoupling analysis of the full-vehicle model is given. The 4WICS control framework based on a hierarchical modeling control is introduced in Sect. 3. In Sect. 4, we present numerical case studies and comparison validation carried out to demonstrate the effectiveness and suitability of the main results obtained. Finally, conclusions are given in Sect. 5.

\section{Dynamic Decoupling Analysis}

\subsection{Before decoupling}

Considering the mutual effects of vertical and lateral forces from the tire, a full-vehicle model with 14 DOFs is adopted and shown in Fig. 1. $x, y$, and $z$ represent the longitudinal, lateral, and vertical directions, respectively. The 14 DOFs are four vertical and four lateral DOFs at four unsprung masses; five DOFs at the center of the sprung mass, i.e., vertical, lateral, pitch angle, roll angle, and yaw angle motions; and one vertical DOF at the driver system.

It is clear that the above traditional full-vehicle model cannot be utilized to obtain the coupling relationships between the full- and quarter-vehicle suspensions. Considering the ratio relationships among the oscillation motions of the full-vehicle body, the sprung mass, and the force supported by each wheel system, the lateral and yaw angle motions at the center of the full sprung mass will emerge. If the relationships between a full-vehicle suspension and quartervehicle suspensions are taken into account, the mutual interactions of full-vehicle vibration motions, the sprung mass, and the force supported by each wheel system should be studied further. The space force analysis of the sprung mass of the full-vehicle suspension is shown in Fig. 2.

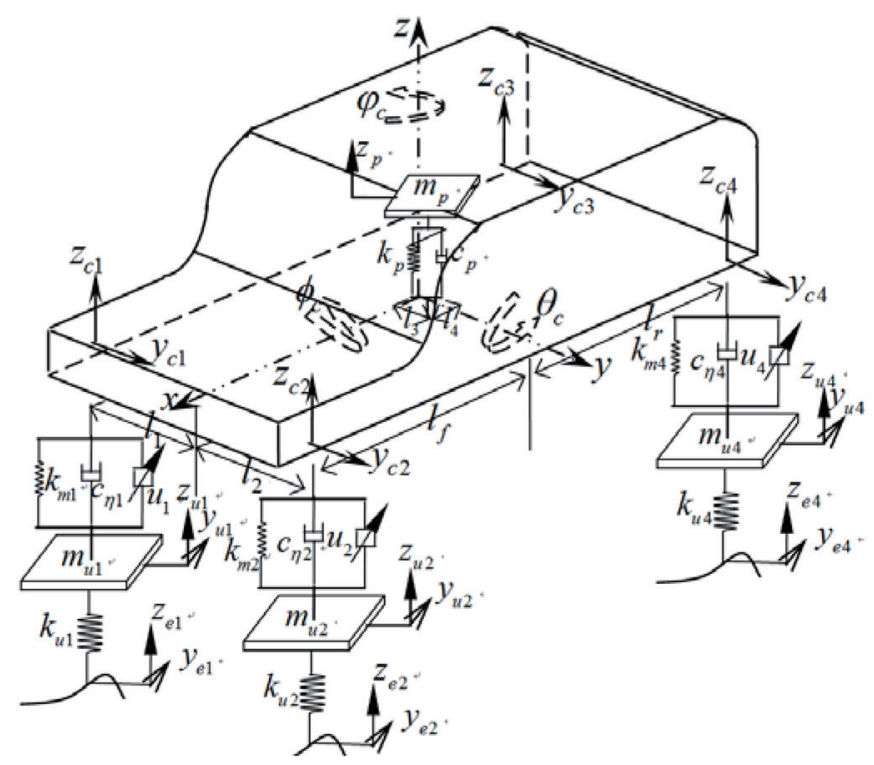

Fig. 1. Vehicle model with 14 DOFs. 


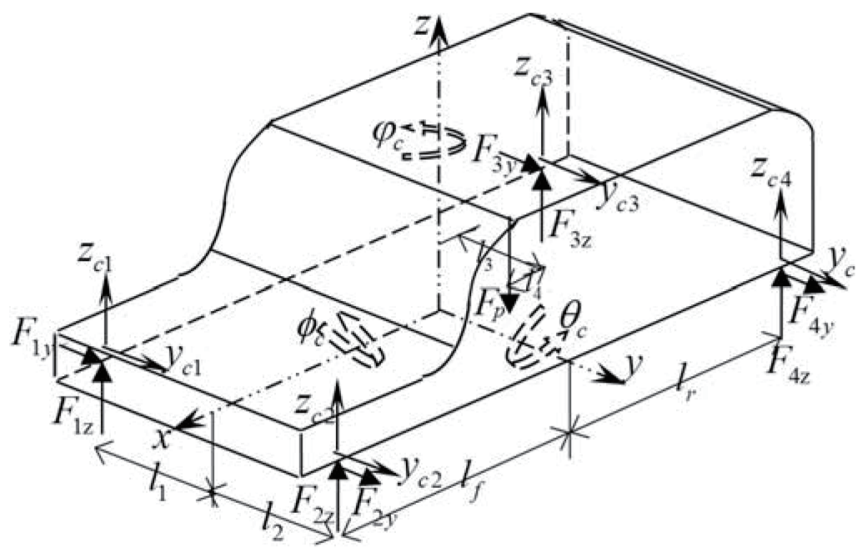

Fig. 2. Force analysis in space for vehicle suspension sprung mass.

In Fig. 2, the spring, damping, and controllable output forces of each wheel system are combined as a concentrated force, and the spring and damping forces of the driver system are also simplified as a concentrated force. The dynamic equations of the full sprung mass, Eqs. (1)-(5), are obtained in terms of the vertical motion $z_{c}$, lateral motion $y_{c}$, pitch angle $\theta_{c}$, roll angle $\phi_{c}$, and yaw angle $\varphi_{c}$ of the mass center.

$$
\begin{gathered}
m_{c} \ddot{z}_{c}=F_{1 z}+F_{2 z}+F_{3 z}+F_{4 z}-F_{p} \\
m_{c} \ddot{y}_{c}=F_{1 y}+F_{2 y}+F_{3 y}+F_{4 y} \\
I_{y} \ddot{\theta}_{c}=-l_{f} F_{1 z}-l_{f} F_{2 z}+l_{r} F_{3 z}+l_{r} F_{4 z}+l_{4} F_{p} \\
I_{x} \ddot{\phi}_{c}=l_{1} F_{1 z}-l_{2} F_{2 z}+l_{1} F_{3 z}-l_{2} F_{4 z}+l_{3} F_{p} \\
I_{z} \ddot{\varphi}_{c}=l_{f} F_{1 y}+l_{f} F_{2 y}-l_{r} F_{3 y}-l_{r} F_{4 y}
\end{gathered}
$$

To reformulate the dynamical equations, the resultant forces $F_{i}, i=1,2,3,4$ are defined as follows. The given coefficients $\lambda_{i j}, i, j=1,2,3,4,5$ are shown in detail in the appendix.

$$
\begin{aligned}
& F_{1}=\lambda_{11} \lambda m_{c} \ddot{z}_{c}+\lambda_{12} \lambda m_{c} \ddot{y}_{c}+\lambda_{13} \lambda I_{y} \ddot{\theta}_{c}+\lambda_{14} \lambda I_{x} \ddot{\phi}_{c}+\lambda_{15} \lambda I_{z} \ddot{\varphi}_{c} \\
& F_{2}=\lambda_{21} \lambda m_{c} \ddot{z}_{c}+\lambda_{22} \lambda m_{c} \ddot{y}_{c}+\lambda_{23} \lambda I_{y} \ddot{\theta}_{c}+\lambda_{24} \lambda I_{x} \ddot{\phi}_{c}+\lambda_{25} \lambda I_{z} \ddot{\varphi}_{c} \\
& F_{3}=\lambda_{31} \lambda m_{c} \ddot{z}_{c}+\lambda_{32} \lambda m_{c} \ddot{y}_{c}+\lambda_{33} \lambda I_{y} \ddot{\theta}_{c}+\lambda_{34} \lambda I_{x} \ddot{\phi}_{c}+\lambda_{35} \lambda I_{z} \ddot{\varphi}_{c}
\end{aligned}
$$




$$
\begin{aligned}
& F_{4}=\lambda_{41} \lambda m_{c} \ddot{z}_{c}+\lambda_{42} \lambda m_{c} \ddot{y}_{c}+\lambda_{43} \lambda I_{y} \ddot{\theta}_{c}+\lambda_{44} \lambda I_{x} \ddot{\phi}_{c}+\lambda_{45} \lambda I_{z} \ddot{\varphi}_{c} \\
& F_{p}=\lambda_{51} \lambda m_{c} \ddot{z}_{c}+\lambda_{52} \lambda m_{c} \ddot{y}_{c}+\lambda_{53} \lambda I_{y} \ddot{\theta}_{c}+\lambda_{54} \lambda I_{x} \ddot{\phi}_{c}+\lambda_{55} \lambda I_{z} \ddot{\varphi}_{c}
\end{aligned}
$$

If the acting forces $F_{i z}, F_{i y}, i=1,2,3,4$ in Eqs. (1)-(5) are treated as two-component forces, the resultant forces $F_{i}, i=1,2,3,4$ given in Eqs. (6)-(10) are replaced by the two-component forces.

Along the $z$ direction, the relationships of vertical displacements between the center of the sprung mass and each corner are obtained from the geometric relationship as follows.

$$
\begin{gathered}
z_{c 1}=z_{c}-l_{f} \theta_{c}+l_{1} \phi_{c} \\
z_{c 2}=z_{c}-l_{f} \theta_{c}-l_{2} \phi_{c} \\
z_{c 3}=z_{c}+l_{r} \theta_{c}+l_{1} \phi_{c} \\
z_{c 4}=z_{c}+l_{r} \theta_{c}-l_{2} \phi_{c} \\
z_{c p}=-z_{c}+l_{4} \theta_{c}+l_{3} \phi_{c}
\end{gathered}
$$

By substituting Eq. (6) into Eq. (1), multiplying by $l_{r}$, then subtracting from Eq. (3), we obtain

$$
m_{c} l_{r} \ddot{z}_{c 1}+m_{c} l_{f} l_{r} \ddot{\theta}_{c}-I_{y} \ddot{\theta}_{c}-m_{c} l_{r} l_{1} \ddot{\phi}_{c}=\left(l_{f}+l_{r}\right) F_{1 z}+\left(l_{f}+l_{r}\right) F_{2 z}-\left(l_{r}+l_{4}\right) F_{p} .
$$

By adding the product of Eq. (16) and $l_{2}$ to the product of Eq. (4) and $\left(l_{f}+l_{r}\right)$, we obtain

$$
\begin{gathered}
m_{c} l_{r} l_{2} \ddot{z}_{c 1}+m_{c} l_{f} l_{r} l_{2} \ddot{\theta}_{c}-I_{y} l_{2} \ddot{\theta}_{c}-m_{c} l_{r} l_{1} l_{2} \ddot{\phi}_{c}+I_{x}\left(l_{f}+l_{r}\right) \ddot{\phi}_{c}= \\
\left(l_{f}+l_{r}\right)\left(l_{1}+l_{2}\right) F_{1 z}+\left(l_{f}+l_{r}\right) l_{1} F_{3 z}-\left(l_{f}+l_{r}\right) l_{2} F_{4 z}-\left(l_{r}+l_{4}\right) l_{2} F_{p}+\left(l_{f}+l_{r}\right) l_{3} F_{p} .
\end{gathered}
$$

By similar procedures, we obtain

$$
\begin{gathered}
m_{c} l_{r} l_{1} \ddot{z}_{c 2}+m_{c} l_{f} l_{r} l_{1} \ddot{\theta}_{c}-I_{y} l_{1} \ddot{\theta}_{c}+m_{c} l_{r} l_{1} l_{2} \ddot{\phi}_{c}-I_{x}\left(l_{f}+l_{r}\right) \ddot{\phi}_{c}= \\
\left(l_{f}+l_{r}\right)\left(l_{1}+l_{2}\right) F_{2 z}-\left(l_{f}+l_{r}\right) l_{1} F_{3 z}+\left(l_{f}+l_{r}\right) l_{2} F_{4 z}-\left(l_{r}+l_{4}\right) l_{1} F_{p}-\left(l_{f}+l_{r}\right) l_{3} F_{p}, \\
m_{c} l_{f} l_{2} \ddot{z}_{c 3}-m_{c} l_{f} l_{r} l_{2} \ddot{\theta}_{c}+I_{y} l_{2} \ddot{\theta}_{c}-m_{c} l_{f} l_{1} l_{2} \ddot{\phi}_{c}+I_{x}\left(l_{f}+l_{r}\right) \ddot{\phi}_{c}= \\
\left(l_{f}+l_{r}\right) l_{1} F_{1 z}-\left(l_{f}+l_{r}\right) l_{2} F_{2 z}+\left(l_{f}+l_{r}\right)\left(l_{1}+l_{2}\right) F_{3 z}-\left(l_{f}-l_{4}\right) l_{2} F_{p}+\left(l_{f}+l_{r}\right) l_{3} F_{p},
\end{gathered}
$$




$$
\begin{gathered}
m_{c} l_{f} l_{1} \ddot{z}_{c 4}-m_{c} l_{f} l_{r} l_{1} \ddot{\theta}_{c}+I_{y} l_{1} \ddot{\theta}_{c}+m_{c} l_{f} l_{1} l_{2} \ddot{\phi}_{c}-I_{x}\left(l_{f}+l_{r}\right) \ddot{\phi}_{c}= \\
-\left(l_{f}+l_{r}\right) l_{1} F_{1 z}+\left(l_{f}+l_{r}\right) l_{2} F_{2 z}+\left(l_{f}+l_{r}\right)\left(l_{1}+l_{2}\right) F_{4 z}-\left(l_{f}-l_{4}\right) l_{1} F_{p}-\left(l_{f}+l_{r}\right) l_{3} F_{p} .
\end{gathered}
$$

Adding Eq. (17) to Eq. (20) gives

$$
m_{c 1} \ddot{z}_{c 1}+m_{c 2} \ddot{z}_{c 2}+m_{c 3} \ddot{z}_{c 3}+m_{c 4} \ddot{z}_{c 4}=F_{1 z}+F_{2 z}+F_{3 z}+F_{4 z}-F_{p},
$$

where

$$
\begin{aligned}
& m_{c 1}=\frac{l_{r} l_{2}}{\left(l_{f}+l_{r}\right)\left(l_{1}+l_{2}\right)} m_{c}, \\
& m_{c 2}=\frac{l_{r} l_{1}}{\left(l_{f}+l_{r}\right)\left(l_{1}+l_{2}\right)} m_{c}, \\
& m_{c 3}=\frac{l_{f} l_{2}}{\left(l_{f}+l_{r}\right)\left(l_{1}+l_{2}\right)} m_{c}, \\
& m_{c 4}=\frac{l_{f} l_{1}}{\left(l_{f}+l_{r}\right)\left(l_{1}+l_{2}\right)} m_{c} .
\end{aligned}
$$

The sum of Eqs. (19) and (16) is multiplied by $l_{r}$, then the result is then subtracted from the sum of Eqs. (17) and (18) multiplied by $l_{f}$, yielding

$$
I_{y}^{h} \ddot{\theta}_{c}=-l_{f} F_{1 z}^{h}-l_{f} F_{2 z}^{h}+l_{r} F_{3 z}^{h}+l_{r} F_{4 z}^{h}+l_{4} F_{p},
$$

where $F_{1 z}^{h}=F_{1 z}-m_{c 1} \ddot{z}_{c 1}, F_{2 z}^{h}=F_{2 z}-m_{c 2} \ddot{z}_{c 2}, F_{3 z}^{h}=F_{3 z}-m_{c 3} \ddot{z}_{c 3}, F_{4 z}^{h}=F_{4 z}-m_{c 4} \ddot{z}_{c 4}$, and $I_{y}^{h}=I_{y}-m_{c} l_{f} l_{r}$.

Similarly, the sum of Eqs. (17) and (19) is multiplied by $l_{1}$, then the result is then subtracted from the sum of Eqs. (18) and (20) multiplied by $l_{2}$, giving

$$
I_{x}^{h} \ddot{\phi}_{c}=l_{1} F_{1 z}^{h h}-l_{2} F_{2 z}^{h h}+l_{1} F_{3 z}^{h h}-l_{2} F_{4 z}^{h h}+l_{3} F_{p},
$$

where $F_{1 z}^{h h}=F_{1 z}-m_{c 1} \ddot{z}_{c 1} / 2, F_{2 z}^{h h}=F_{2 z}-m_{c 2} \ddot{z}_{c 2} / 2, F_{3 z}^{h h}=F_{3 z}-m_{c 3} \ddot{z}_{c 3} / 2, F_{4 z}^{h h}=F_{4 z}-m_{c 4} \ddot{z}_{c 4} / 2$, and $I_{x}^{h}=I_{x}-m_{c} l_{1} l_{2} / 2$.

Along the $y$ direction, the relationships of lateral displacements between the center of the sprung mass and each corner can be obtained from the geometric relationship as follows. 


$$
\begin{aligned}
& y_{c 1}=y_{c}+l_{f} \varphi_{c}+l_{1} \phi_{c} \\
& y_{c 2}=y_{c}+l_{f} \varphi_{c}-l_{2} \phi_{c} \\
& y_{c 3}=y_{c}-l_{r} \varphi_{c}+l_{1} \phi_{c} \\
& y_{c 4}=y_{c}-l_{r} \varphi_{c}-l_{2} \phi_{c}
\end{aligned}
$$

Substituting Eqs. (28)-(31) into Eqs. (2)-(5), respectively, gives

$$
\begin{gathered}
m_{c 1} \ddot{y}_{c 1}+m_{c 2} \ddot{y}_{c 2}+m_{c 3} \ddot{y}_{c 3}+m_{c 4} \ddot{y}_{c 4}=F_{1 y}+F_{2 y}+F_{3 y}+F_{4 y}, \\
I_{z}^{h} \ddot{\varphi}_{c}=l_{f} F_{1 y}^{h}+l_{f} F_{2 y}^{h}-l_{r} F_{3 y}^{h}-l_{r} F_{4 y}^{h},
\end{gathered}
$$

where $F_{1 y}^{h}=F_{1 y}-m_{c 1} \ddot{y}_{c 1}, F_{2 y}^{h}=F_{2 y}-m_{c 2} \ddot{y}_{c 2}, F_{3 y}^{h}=F_{3 y}-m_{c 3} \ddot{y}_{c 3}, F_{4 y}^{h}=F_{4 y}-m_{c 4} \ddot{y}_{c 4}$, and $I_{z}^{h}=I_{z}-m_{c} l_{f} l_{r}$.

The derived results indicate that Eqs. (21), (26), (27), (32), and (33) have similar forms to Eqs. (1)-(5), respectively. However, the original sprung mass of a full vehicle is substituted by four sprung masses of quarter vehicles. Moreover, if $l_{f}=l_{r}$ and $l_{1}=l_{2}$, the mathematical expressions for the decoupling sprung masses show that $m_{c 1}, m_{c 2}, m_{c 3}$, and $m_{c 4}$ are a quarter of $m_{c}$. If $l_{f} \neq l_{r}$ and $l_{1} \neq l_{2}$, the ratio relationships between a full vehicle and four quarter vehicles can still be determined. A full vehicle could be seen as a combination of four quarter vehicles. The five motions of the full-vehicle sprung mass center are equivalent to the four vertical motions and one lateral motion of the quarter-vehicle suspension sprung masses. Therefore, we suppose that without the connective coupling actions, a full sprung mass can be decomposed into four sprung masses of quarter vehicles.

\subsection{After decoupling}

Owing to the effectiveness of a continuous sprung mass, each quarter-vehicle sprung mass is incorporated into internal coupling actions. Considering that each quarter-vehicle sprung mass is independent and lacks the limitations of other adjacent sprung masses, the vertical and lateral positions of each decoupled quarter-vehicle sprung mass can be changed. In the following process of removing adjacent constraints, the vertical and lateral displacement variables of each sprung mass supported by each wheel system are $\Delta z_{c i}$ and $\Delta z_{c i}$, and the vertical and lateral displacement variables of each sprung mass without restrictions are $z_{i}$ and $y_{i}$, respectively. In the $z$ direction, the vertical relationships of the sprung mass displacement of wheel 1 are given as follows. 


$$
\begin{gathered}
m_{c 1} \ddot{z}_{1}=F_{1 z}-m_{c 1} \Delta \ddot{z}_{c 1} \\
\Delta z_{c 1}=z_{1}-z_{c 1}
\end{gathered}
$$

By using the geometric relationship of Eq. (11), we obtain

$$
\Delta \ddot{z}_{c 1}=\frac{\cos \gamma_{1}}{2 m_{c 1}} F_{1}-\frac{1}{2} \ddot{z}_{c}+\frac{l_{f}}{2} \ddot{\theta}_{c}-\frac{l_{1}}{2} \ddot{\phi}_{c} .
$$

The vertical relationships of the sprung mass displacements of the other wheel systems are shown as follows.

$$
\begin{gathered}
m_{c 2} \ddot{z}_{2}=F_{2 z}-m_{c 2} \Delta \ddot{z}_{c 2} \\
\Delta z_{c 2}=z_{2}-z_{c 2} \\
m_{c 3} \ddot{z}_{3}=F_{3 z}+m_{c 3} \Delta \ddot{z}_{c 3} \\
\Delta z_{c 3}=z_{c 3}-z_{3} \\
m_{c 4} \ddot{z}_{4}=F_{4 z}+m_{c 4} \Delta \ddot{z}_{c 4} \\
\Delta z_{c 4}=z_{c 4}-z_{4}
\end{gathered}
$$

Similarly, we obtain

$$
\begin{gathered}
\Delta \ddot{z}_{c 2}=\frac{\cos \gamma_{2}}{2 m_{c 2}} F_{2}-\frac{1}{2} \ddot{z}_{c}+\frac{l_{f}}{2} \ddot{\theta}_{c}+\frac{l_{2}}{2} \ddot{\phi}_{c}, \\
\Delta \ddot{z}_{c 3}=-\frac{\cos \gamma_{3}}{2 m_{c 3}} F_{3}+\frac{1}{2} \ddot{z}_{c}+\frac{l_{r}}{2} \ddot{\theta}_{c}+\frac{l_{1}}{2} \ddot{\phi}_{c}, \\
\Delta \ddot{z}_{c 4}=-\frac{\cos \gamma_{4}}{2 m_{c 4}} F_{4}+\frac{1}{2} \ddot{z}_{c}+\frac{l_{r}}{2} \ddot{\theta}_{c}-\frac{l_{2}}{2} \ddot{\phi}_{c} .
\end{gathered}
$$

In the $y$ direction, the lateral relationships of the sprung mass displacement of wheel 1 are given as follows.

$$
m_{c 1} \ddot{y}_{1}=F_{1 y}-m_{c 1} \Delta \ddot{y}_{c 1}
$$




$$
\Delta y_{c 1}=y_{1}-y_{c 1}
$$

By using the geometric relationship of Eq. (28), we obtain

$$
\begin{gathered}
\Delta \ddot{y}_{c 1}=\frac{\sin \gamma_{1}}{2 m_{c 1}} F_{1}-\frac{1}{2} \ddot{y}_{c}-\frac{l_{f}}{2} \ddot{\varphi}_{c}-\frac{l_{1}}{2} \ddot{\phi}_{c}, \\
\Delta \ddot{y}_{c 2}=-\frac{\sin \gamma_{2}}{2 m_{c 2}} F_{2}+\frac{1}{2} \ddot{y}_{c}+\frac{l_{f}}{2} \ddot{\varphi}_{c}-\frac{l_{2}}{2} \ddot{\phi}_{c}, \\
\Delta \ddot{y}_{c 3}=\frac{\sin \gamma_{3}}{2 m_{c 3}} F_{3}-\frac{1}{2} \ddot{y}_{c}+\frac{l_{r}}{2} \ddot{\varphi}_{c}-\frac{l_{1}}{2} \ddot{\phi}_{c}, \\
\Delta \ddot{y}_{c 4}=-\frac{\sin \gamma_{4}}{2 m_{c 4}} F_{4}+\frac{1}{2} \ddot{y}_{c}-\frac{l_{r}}{2} \ddot{\varphi}_{c}-\frac{l_{2}}{2} \ddot{\phi}_{c} .
\end{gathered}
$$

Accordingly, the position variations of unsprung masses should be considered. The ordered vertical and lateral displacements of each unsprung mass are assigned the variables $\Delta z_{u i}$ and $\Delta y_{u i}$ in the process of removing adjacent constraints, and the vertical and lateral displacements of each unsprung mass after decoupling are assigned the variables $z_{u i}^{*}$ and $y_{u i}^{*}$, respectively. The relationships of the vertical and lateral directions of the unsprung mass displacements of wheel 1 are given as follows.

$$
\begin{aligned}
& \Delta z_{u 1}=z_{u 1}^{*}-z_{u 1} \\
& \Delta y_{u 1}=y_{u 1}^{*}-y_{u 1}
\end{aligned}
$$

The vertical dynamic balance equations of the unsprung mass displacement for wheel 1 before and after decoupling are as follows.

$$
\begin{gathered}
m_{u 1} \ddot{z}_{u 1}=k_{m 1}\left(z_{c 1}-z_{u 1}\right)+c_{\eta 1}\left(\dot{z}_{c 1}-\dot{z}_{u 1}\right)-k_{u 1}\left(z_{u 1}-z_{e 1}\right)-u_{z 1} \\
m_{u 1} \ddot{z}_{u 1}^{*}=k_{m 1}\left(z_{1}-z_{u 1}^{*}\right)+c_{\eta 1}\left(\dot{z}_{1}-\dot{z}_{u 1}^{*}\right)-k_{u 1}\left(z_{u 1}^{*}-z_{e 1}\right)-u_{z 1}
\end{gathered}
$$

Equations (54) and (55) are subtracted from each other and substituted into Eq. (52), yielding

$$
m_{u 1} \Delta \ddot{z}_{u 1}+c_{\eta 1} \Delta \dot{z}_{c 1}-\left(k_{u 1}+k_{m 1}\right) \Delta z_{u 1}=c_{\eta 1} \Delta \dot{z}_{u 1}-k_{m 1} \Delta z_{c 1}
$$


The lateral motion results of the unsprung mass for wheel 1 can be obtained similarly to the vertical ones.

$$
m_{u 1} \Delta \ddot{y}_{u 1}+c_{\eta 1} \Delta \dot{y}_{u 1}+\left(k_{u 1}+k_{m 1}\right) \Delta y_{u 1}=k_{m 1} \Delta y_{c 1}+c_{\eta 1} \Delta \dot{y}_{c 1}
$$

Similarly, the vertical and lateral motion results of other unsprung masses are obtained as follows.

$$
\begin{gathered}
m_{u 2} \Delta \ddot{z}_{u 2}+c_{\eta 2} \Delta \dot{z}_{c 2}-\left(k_{u 2}+k_{m 2}\right) \Delta z_{u 2}=c_{\eta 2} \Delta \dot{z}_{u 2}-k_{m 2} \Delta z_{c 2} \\
m_{u 2} \Delta \ddot{y}_{u 2}+c_{\eta 2} \Delta \dot{y}_{c 2}-\left(k_{u 2}+k_{m 2}\right) \Delta y_{u 2}=c_{\eta 2} \Delta \dot{y}_{u 2}-k_{m 2} \Delta y_{c 2} \\
m_{u 3} \Delta \ddot{z}_{u 3}+c_{\eta 3} \Delta \dot{z}_{u 3}+\left(k_{m 3}+k_{u 3}\right) \Delta z_{u 3}=k_{m 3} \Delta z_{c 3}+c_{\eta 3} \Delta \dot{z}_{c 3} \\
m_{u 3} \Delta \ddot{y}_{u 3}+c_{\eta 3} \Delta \dot{y}_{u 3}+\left(k_{u 3}+k_{m 3}\right) \Delta y_{u 3}=k_{m 3} \Delta y_{c 3}+c_{\eta 3} \Delta \dot{y}_{c 3} \\
m_{u 4} \Delta \ddot{z}_{u 4}+c_{\eta 4} \Delta \dot{z}_{u 4}+\left(k_{m 4}+k_{u 4}\right) \Delta z_{u 4}=k_{m 4} \Delta z_{c 4}+c_{\eta 4} \Delta \dot{z}_{c 4} \\
m_{u 4} \Delta \ddot{y}_{u 4}+c_{\eta 4} \Delta \dot{y}_{u 4}+\left(k_{m 4}+k_{u 4}\right) \Delta y_{u 4}=k_{m 4} \Delta y_{c 4}+c_{\eta 4} \Delta \dot{y}_{c 4}
\end{gathered}
$$

According to the above deductions of sprung and unsprung masses in the decoupling process, a full-vehicle model under vertical and lateral road excitations is theoretically treated as a combination of four parallel quarter-vehicle models. Furthermore, each quarter-vehicle model can be decomposed into vertical and lateral quarter-vehicle suspension models with two DOFs. The above treatment is not only helpful to future quantitative studies of theory and testing, but also forms a new parallel control model of quarter-vehicle systems.

\section{4WICS Control Framework Based on Hierarchical Modeling Method}

Since a full-vehicle model is transformed into four parallel quarter-vehicle models, the vibration control for a full vehicle is turned into parallel controls of four quarter vehicles. The 4WICS control framework based on a hierarchical modeling method can be developed in this way. The hierarchical modeling method in this work is the use of a double-layer model based on the process of decoupling a full vehicle. The model contains a center control layer and four local control layers. The center control layer is constructed using an algorithm based on the coupling relationships between a full vehicle and four quarter vehicles. The goal of the center control layer is to stabilize five control variables, namely, the vertical, lateral, pitch angle, roll angle, and yaw angle vibrations of the whole vehicle system, to ensure ride comfort and driving 
stability. Four local control layers correspond to four parallel quarter vehicles. The goal of each local control is to develop a certain control strategy to make the actual movement of each quarter-vehicle sprung mass approach the pre-estimated value. The hierarchical modeling method is realized by a model reference adaptive control mechanism. The relationships between the center control and the local controls are shown in Fig. 3.

The operating procedures for the center control layer in the hierarchical modeling method are as follows. (A) The five pre-estimated values of a full-vehicle sprung mass center, namely, the vertical, lateral, pitch angle, roll angle, and yaw angle accelerations, are determined according to the vertical and lateral excitations. The given range of the above pre-estimated values will not statistically exceed $99.7 \%$ of their minimum values while satisfying the requirements of

$$
\begin{aligned}
& \ddot{z}_{c} \leq 1 / 3 \sigma_{1}, \\
& \ddot{y}_{c} \leq 1 / 3 \sigma_{2}, \\
& \ddot{\theta}_{c} \leq 1 / 3 \sigma_{3}, \\
& \ddot{\phi}_{c} \leq 1 / 3 \sigma_{4}, \\
& \ddot{\varphi}_{c} \leq 1 / 3 \sigma_{5},
\end{aligned}
$$

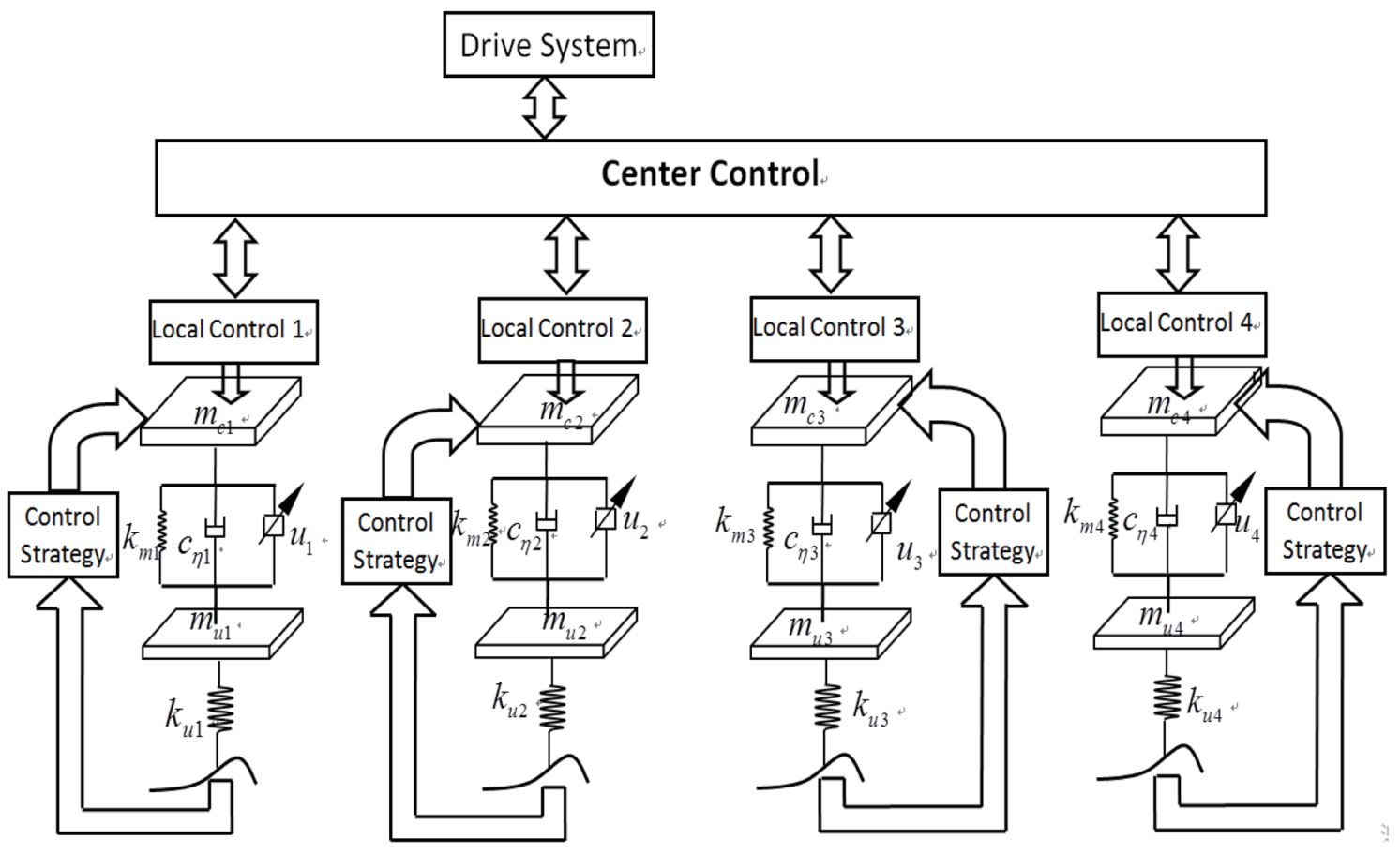

Fig. 3. Hierarchical modeling control framework. 
where $\sigma_{1}, \sigma_{3}, \sigma_{3}, \sigma_{4}$, and $\sigma_{5}$ are the intermediate variables formulated according to the vertical and lateral excitations as follows.

$$
\begin{gathered}
\sigma_{1}=0.6\left(z_{e 1}+z_{e 2}+z_{e 3}+z_{e 4}\right) /\left(4 t_{s}^{2}\right) \\
\sigma_{2}=0.6\left(y_{e 1}+y_{e 2}+y_{e 3}+y_{e 4}\right) /\left(4 t_{s}^{2}\right) \\
\sigma_{3}=0.6\left\{\tan ^{-1}\left[\left(z_{e 3}+z_{e 4}\right)-\left(z_{e 1}+z_{e 2}\right)\right] /\left[2\left(l_{f}+l_{r}\right)\right]\right\} / t_{s}^{2} \\
\sigma_{4}=0.6\left\{\tan ^{-1}\left[l_{f}\left(z_{e 4}-z_{e 3}\right)+l_{r}\left(z_{e 2}-z_{e 1}\right)\right] /\left[\left(l_{f}+l_{r}\right)\left(l_{1}+l_{2}\right)\right]\right\} / t_{s}^{2} \\
\sigma_{5}=0.6\left\{\tan ^{-1}\left[\left(y_{e 2}-y_{e 1}\right)-\left(y_{e 4}-y_{e 3}\right)\right] /\left(l_{f}+l_{r}\right)\right\} / t_{s}^{2}
\end{gathered}
$$

(B) The pre-estimated values of $F_{1}, F_{2}, F_{3}, F_{4}$, and $F_{5}$ are obtained from the values of $\ddot{z}_{c}, \ddot{y}_{c}, \ddot{\theta}_{c}$, $\ddot{\phi}_{c}$ and $\ddot{\varphi}_{c}$ using Eqs. (6)-(10). According to Eq. (36), Eqs. (43)-(45), and Eqs. (48)-(51), the pre-estimated values from $\Delta \ddot{z}_{c 1}$ to $\Delta \ddot{z}_{c 4}$ and from $\Delta \ddot{y}_{c 1}$ to $\Delta \ddot{y}_{c 4}$ can be calculated. The preestimated values from $\ddot{z}_{c 1}$ to $\ddot{z}_{c 4}$ and from $\ddot{y}_{c 1}$ to $\ddot{y}_{c 4}$ are also ascertained using Eqs. (11)-(14) and Eqs. (28)-(31), respectively. On the basis of the above results, the pre-estimated values of $\ddot{y}_{i}, \ddot{z}_{i}, i=1,2,3,4 \ddot{y}_{4}$ can be obtained. (C) The pre-estimated values are transferred to local control layers, from $\ddot{z}_{1}$ to $\ddot{z}_{4}$ and from $\ddot{y}_{1}$ to $\ddot{y}_{4}$. (D) The actual motion values of the four quarter vehicles are acquired. (E) The reverse process is carried out. The actual motions of the four corners from $\Delta \ddot{z}_{c 1}$ to $\Delta \ddot{z}_{c 4}$ and from $\Delta \ddot{y}_{c 1}$ to $\Delta \ddot{y}_{c 4}$ are provided. The actual motions of a full-vehicle sprung mass center with vertical, lateral, pitch angle, roll angle, and yaw angle accelerations can be obtained.

The operating procedures for each local control layer in the hierarchical modeling method are as follows. (a) The pre-estimated vertical and lateral sprung mass accelerations from the center control layer are obtained. (b) Because most vehicle dampers and springs are mounted vertically, the vertical vibration is controlled. When the pre-estimated movement of the sprung mass is determined, the vertical control volume $U_{Z}$ can be calculated using a certain control strategy and a state-space equation of a quarter-vehicle suspension model with 2 DOFs. Then, the required controllable force can be exerted. Consequently, the actual accelerations of the sprung and unsprung masses are acquired. (c) The error between the theoretical and actual control volumes in the current round is calculated and compensated for in the next round. (d) The actual vibration excitation values of the sprung mass to the center control layer are provided. 


\section{Results of Case Studies and Discussion}

In this section, we report case studies performed to verify the effectiveness of the 4WICS based on the hierarchical modeling method for a full-vehicle system. Table 1 shows the vehicle system parameters used for simulation and comparison with the traditional full-vehicle suspension with the driver system reported previously. ${ }^{(29,30)}$

Because most vehicle dampers and springs are vertically mounted, the vertical impacts of the road are minimized. The vertical, pitch angle, and roll angle accelerations of the full vehicle are selected to be the performance indexes. Moreover, the lateral excitations generated by the tire effect usually have small values, whereas the lateral and yaw angle accelerations of the full vehicle remain to some extent. Thus, the lateral excitations are utilized to obtain the vertical characteristics of the full suspension because they are not notable when the vehicle is moving in a uniform, straight trajectory. Under the small-angle condition, $\sin \gamma_{i}=\gamma_{i}, \cos \gamma_{i}=1, \gamma_{i}(i=1,2,3,4)$, the local control should only execute vertical control for the suspension model with 2 DOFs. The computation process for 4WICS based on the hierarchical modeling method is presented in Fig. 4.

The simulation results of cases tested for 4WICS based on hierarchical modeling control are compared with the results in Ref. 30. Some test settings and options are similarly adopted in advance. First, four active control dampers are employed in four-wheel systems. The suspension travel range is $\pm 0.1 \mathrm{~m}$. The tire deflection range is from -0.1 to $0.1 \mathrm{~m}$. Some sophisticated blocks of the control strategies in the Matlab software environment are applied. The robust P-systems and linear quadratic Gaussian (LQG) algorithm are operated in the front and rear local layer systems, respectively.

The sampling time for road excitation is $0.02 \mathrm{~s}$ and the continuous waves generated by road roughness are used for the computation of the full-vehicle suspension vibration. However, owing to different calculation times required for finishing a round control in the hierarchical modeling control method, the CPU time for the current round control is combined with road continuous waves to obtain the next data for road roughness in the interpolation for the next round control. The computation is carried out on a computer with an $8 \mathrm{GHz} \mathrm{CPU}$ and $16 \mathrm{~GB}$ memory, and programmed in the environment of the 18th edition of Matlab software. Lateral

Table 1

System parameters of a vehicle system.

\begin{tabular}{ccccccc}
\hline Name & Value & Unit & & Name & Value & Unit \\
\cline { 1 - 3 } \cline { 5 - 7 }$m_{p}$ & 75 & $\mathrm{~kg}$ & & $c_{\eta 3}, c_{\eta 4}$ & 1620 & $\mathrm{~N} \cdot \mathrm{s} / \mathrm{m}$ \\
$c_{p}$ & 12829 & $\mathrm{~N} \cdot \mathrm{s} / \mathrm{m}$ & & $k_{m 1}, k_{m 2}$ & 19960 & $\mathrm{~N} / \mathrm{m}$ \\
$k_{p}$ & 8791 & $\mathrm{~N} / \mathrm{m}$ & & $k_{m 3}, k_{m} 4$ & 17500 & $\mathrm{~N} / \mathrm{m}$ \\
$m_{c}$ & 730 & $\mathrm{~kg}$ & & $k_{u 1}, k_{u 2}$ & 175500 & $\mathrm{~N} / \mathrm{m}$ \\
$I_{y}$ & 1230 & $\mathrm{~kg} \cdot \mathrm{m}^{2}$ & & $k_{u 1}, k_{u 2}$ & 175500 & $\mathrm{~N} / \mathrm{m}$ \\
$I_{x}$ & 1230 & $\mathrm{~kg} \cdot \mathrm{m}^{2}$ & & $l_{f}$ & 1.011 & $\mathrm{~m}$ \\
$I_{z}$ & 460 & $\mathrm{~kg} \cdot \mathrm{m}^{2}$ & & $l_{r}$ & 1.803 & $\mathrm{~m}$ \\
$m_{u 1}, m_{u 2}$ & 40 & $\mathrm{~kg}$ & & $l_{1}, l_{2}$ & 0.761 & $\mathrm{~m}$ \\
$m_{u 3}, m_{u 2}$ & 35.5 & $\mathrm{~kg}$ & $l_{3}$ & 0.755 & $\mathrm{~m}$ \\
$c_{\eta 1}, c_{\eta 2}$ & 1290 & $\mathrm{~N} \cdot \mathrm{s} / \mathrm{m}$ & & $l_{4}$ & 0.755 & $\mathrm{~m}$ \\
\hline
\end{tabular}




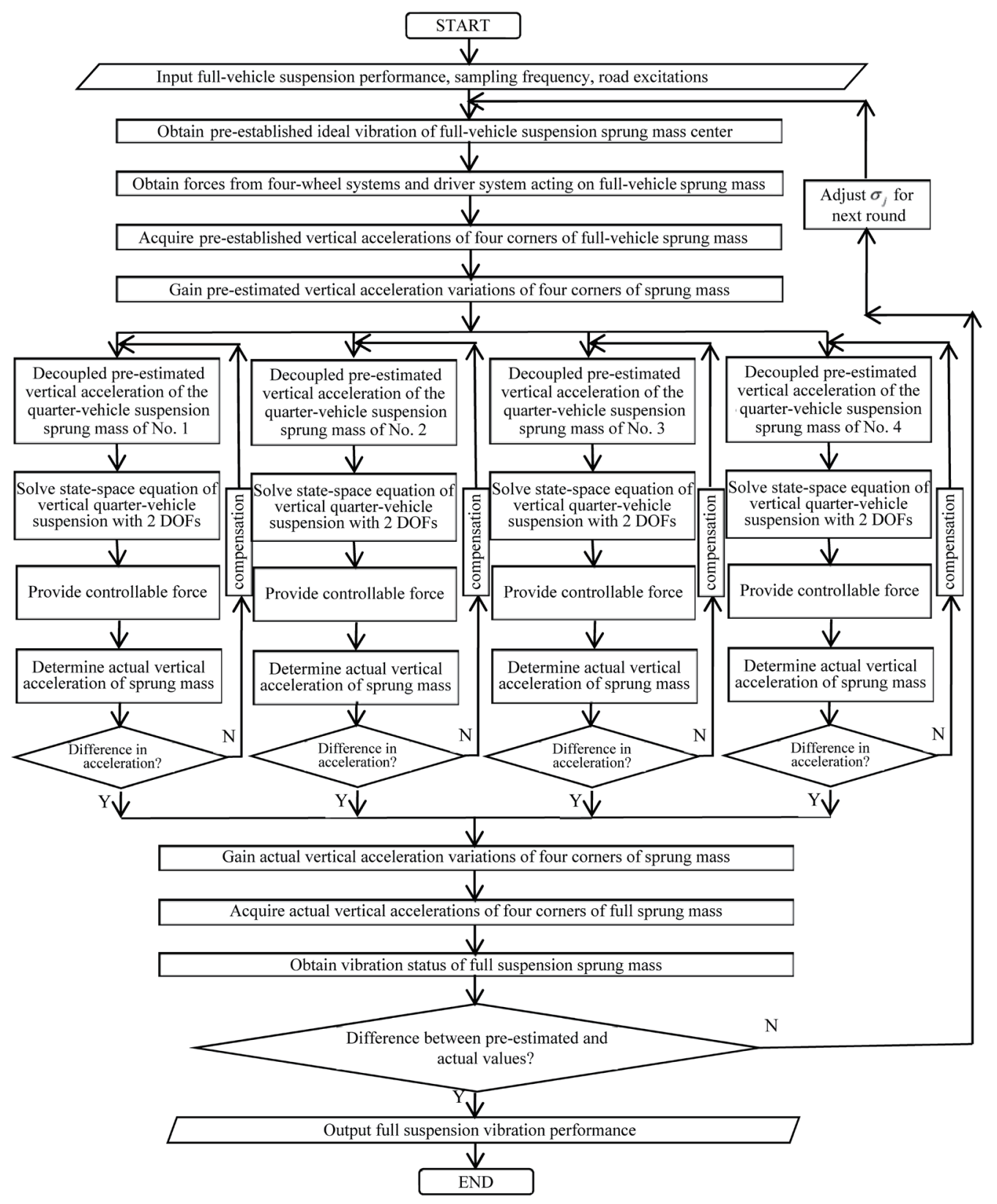

Fig. 4. Computation process for hierarchical modeling control.

tire forces are given by the tire magic formula. Since the lateral tire forces are smaller than vertical forces and hardly decrease owing to the high stiffness and weak damper in the lateral direction, the lateral forces $F_{i y}$ are substituted with the lateral tire forces to simplify the computation. In the following, the case studies are divided into three categories, namely, bump response, road response, and computation time, to verify the performance of 4WICS on the basis of hierarchical modeling control. 


\subsection{Comparison of bump response}

To investigate the transient response of a car passing a bump under the active control of 4WICS, vertical sinusoidal pulse excitations with amplitudes of $0.095 \mathrm{~m}$ are adopted. The vehicle speed is $30 \mathrm{~km} / \mathrm{h}$. The motion responses of a full vehicle through the obstacle are presented in Figs. 5-8. In these figures, the continuous and dotted lines respectively correspond to 4 WICS and traditional active vibration control results.

Figures 5-8 show that the vibration responses of accelerations for a whole car moving under the control of 4WICS are lower than those under the traditional active vibration control when the full suspension encounters sinusoidal pulse excitation. On the other hand, the response speed of the 4WICS control is significantly higher and returns to the steady state more rapidly than under the traditional active vibration. The entire period of sinusoidal bounces is $0.4 \mathrm{~s}$. The results for the 4WICS control show that the vibration response returns to the steady state in $0.5 \mathrm{~s}$. The recovery rate is 1.25 , which is comparable to 1.375 in Ref. 30, indicating that 4WICS shows a slightly higher performance. Owing to the shortened calculation time for control variables and the higher response speed during the parallel running of four controllable local layers under 4WICS control, the vehicle ride comfort and driving stability will be improved rapidly when encountering obstacles.

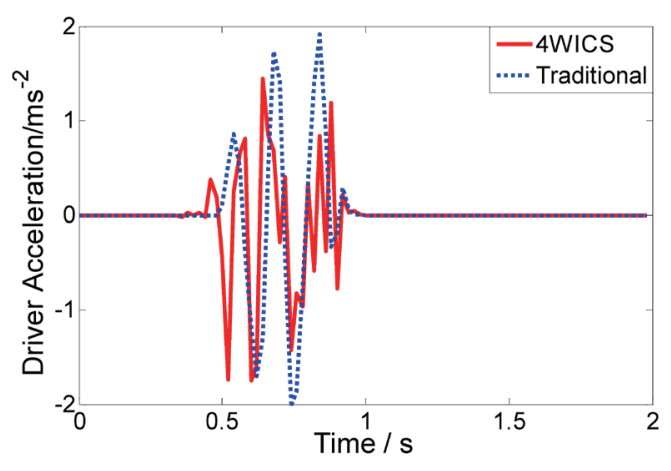

Fig. 5. (Color online) Driver acceleration.

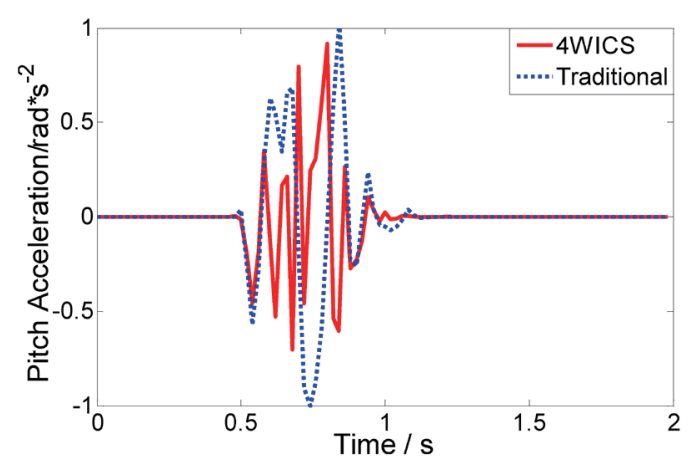

Fig. 7. (Color online) Pitch acceleration.

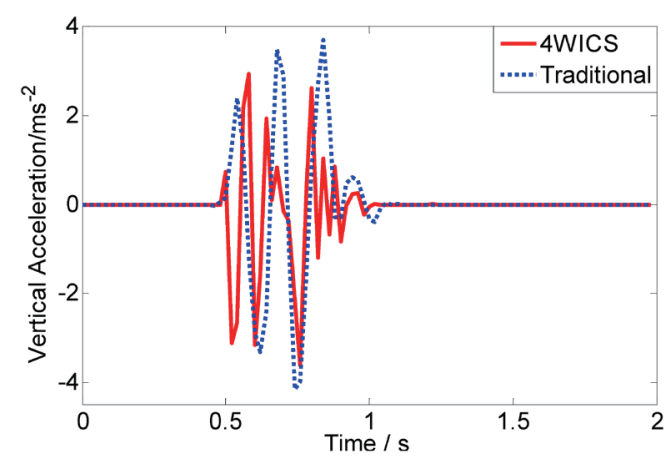

Fig. 6. (Color online) Bounce acceleration.

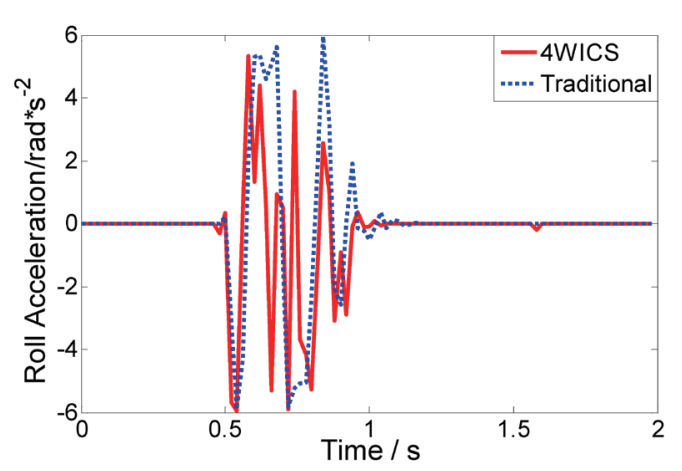

Fig. 8. (Color online) Roll acceleration. 


\subsection{Comparison of road response}

The excitation on a C-grade road is considered. Du et al. used the power spectral density (PSD) expression for road disturbance generated in the time domain. ${ }^{(29)}$ Figure 9 shows the C-grade road roughness under each wheel at a vehicle speed of $60 \mathrm{~km} / \mathrm{h}$.

Spectral analysis was carried out to compare the root-mean-square (RMS) acceleration between the 4WICS control and the traditional active vibration control, the results of which are shown in Figs. 10-13. In these figures, the continuous and dotted lines correspond to the 4WICS and traditional active vibration control results, respectively.

Figure 10 shows peak accelerations of 0.235 and $0.316 \mathrm{~m} / \mathrm{s}^{2}$ at 1.05 and $1.2 \mathrm{~Hz}$ for the 4WICS and traditional active vibration controls, respectively. The decrease in the driver's vertical acceleration peak is approximately $25.6 \%$ for the 4 WICS control compared with the traditional active vibration control. Additionally, the second peak accelerations for the 4WICS and traditional active vibration controls are 0.2 and $0.23 \mathrm{~m} / \mathrm{s}^{2}$, which occurred at 5.6 and $5.8 \mathrm{~Hz}$, respectively. The decrease is about $13.0 \%$ for the $4 \mathrm{WICS}$ control compared with the traditional active vibration control. Figure 11 shows the vertical accelerations of the sprung mass center at different frequencies. The acceleration peaks are 0.50 and $0.44 \mathrm{~m} / \mathrm{s}^{2}$ for the traditional active vibration control and 0.40 and $0.32 \mathrm{~m} / \mathrm{s}^{2}$ for the 4WICS control at 0.9 and $1.2 \mathrm{~Hz}$, respectively. Note that the acceleration for the 4WICS control is higher than that for the traditional active vibration control at $6 \mathrm{~Hz}$. Figure 12 shows that the pitch acceleration peak for the 4WICS control is lower than that for the traditional active vibration control in the frequency range from 1 to $6.5 \mathrm{~Hz}$. Figure 13 shows that the maximum roll acceleration is reduced by $17 \%$ at $1.2 \mathrm{~Hz}$, $58.2 \%$ at $1.9 \mathrm{~Hz}, 36 \%$ at $2.5 \mathrm{~Hz}, 12.4 \%$ at $4.5 \mathrm{~Hz}$, and $90.8 \%$ at $5.8 \mathrm{~Hz}$.
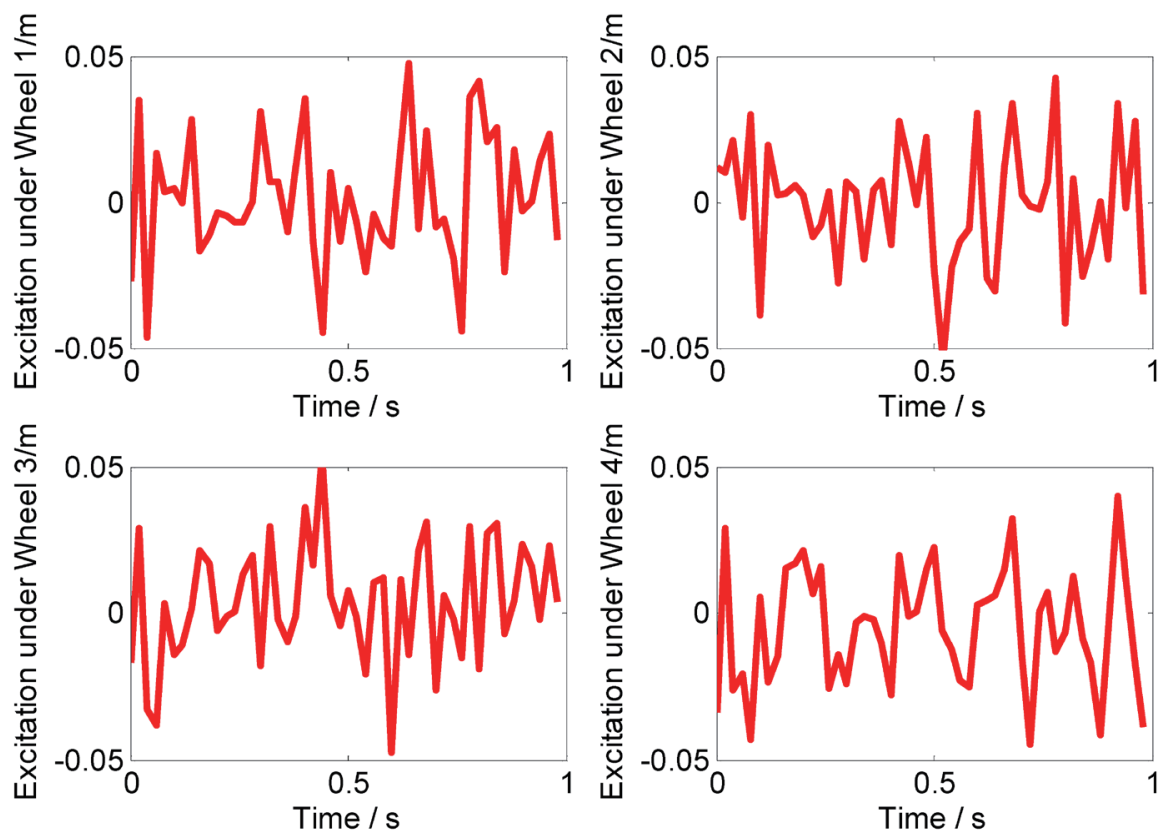

Fig. 9. (Color online) C-grade road roughness. 


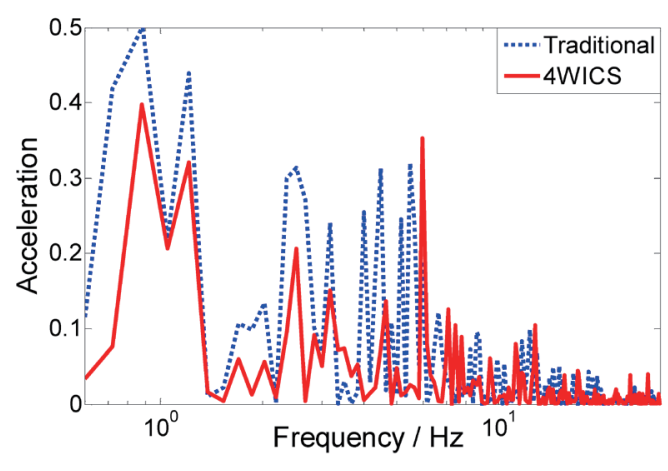

Fig. 10. (Color online) Driver acceleration.

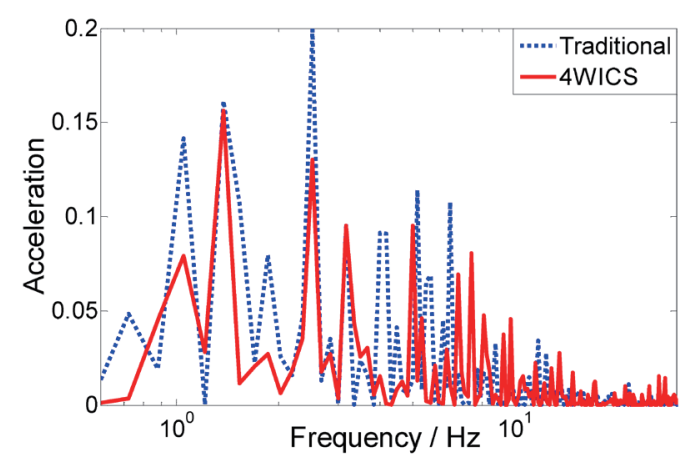

Fig. 12. (Color online) Pitch acceleration.

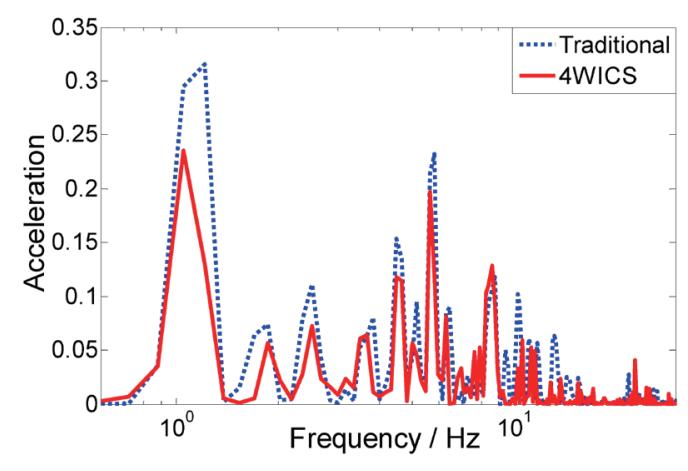

Fig. 11. (Color online) Vertical acceleration.

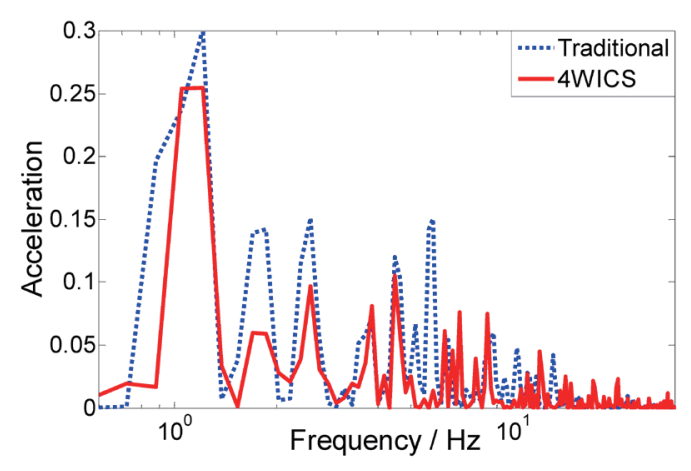

Fig. 13. (Color online) Roll acceleration.

Generally, the RMS accelerations in the frequency range in which humans are sensitive $(1-10 \mathrm{~Hz})$, have mostly been decreased. Although some 4WICS control values at integer frequencies are higher than the traditional control values, the absolute values at these frequencies remain smaller than the uncontrolled values. ${ }^{(29,30)}$ The integer frequencies are shown in Table 2, where Comparison (\%) is defined by (4WICS - Traditional)/Traditional.

\subsection{Comparison of computation time}

The CPU computation times of the 4WICS and traditional active controls for a whole vehicle are investigated. Two hundred road excitation data points are used for computation and comparison. The total time of 200 cycles when employing 4WICS is 1.163 and that for the traditional method is $3.954 \mathrm{~s}$. Thus, the computation time is reduced by $70.6 \%$ for the 4WICS control compared with the traditional control. Owing to the simplification of the full-vehicle model, the computations for full-vehicle traditional control involve four parallel computations for the local layers. A short computation time means a rapid control response. It is also revealed that a perfect control system could be realized by collecting more detailed road information. In addition, the parallel control of four quarter suspensions is particularly suitable for an in-wheel motor-driven electric vehicle and four-wheel-independent-controllable vehicles. Therefore, 4WICS control based on the hierarchical modeling method is useful for improving the performance of a full-vehicle suspension. 
Table 2

RMS values at sensitive frequency points.

\begin{tabular}{|c|c|c|c|c|c|}
\hline & & Driver & Vertical & Pitch & Roll \\
\hline \multirow{3}{*}{$1 \mathrm{~Hz}$} & 4WICS & 0.1850 & 0.2597 & 0.0693 & 0.1855 \\
\hline & Traditional & 0.1657 & 0.2967 & 0.1062 & 0.2260 \\
\hline & Comparison \% & $58.80 \uparrow$ & $12.47 \downarrow$ & $34.75 \downarrow$ & $17.92 \downarrow$ \\
\hline \multirow{3}{*}{$2 \mathrm{~Hz}$} & 4WICS & 0.0275 & 0.0475 & 0.0087 & 0.0337 \\
\hline & Traditional & 0.0198 & 0.1275 & 0.0358 & 0.0314 \\
\hline & Comparison \% & $38.89 \uparrow$ & $62.75 \downarrow$ & $75.70 \downarrow$ & $7.325 \uparrow$ \\
\hline \multirow{3}{*}{$3 \mathrm{~Hz}$} & 4WICS & 0.0097 & 0.0542 & 0.0055 & 0.0054 \\
\hline & Traditional & 0.0023 & 0.0718 & 0.0039 & 0.0017 \\
\hline & Comparison \% & $321.7 \uparrow$ & $24.51 \downarrow$ & $52.78 \uparrow$ & $217.7 \uparrow$ \\
\hline \multirow{3}{*}{$4 \mathrm{~Hz}$} & 4WICS & 0.0074 & 0.0510 & 0.0151 & 0.0036 \\
\hline & Traditional & 0.0046 & 0.2326 & 0.0915 & 0.0040 \\
\hline & Comparison \% & $60.87 \uparrow$ & $78.07 \downarrow$ & $83.50 \downarrow$ & $10.00 \downarrow$ \\
\hline \multirow{3}{*}{$5 \mathrm{~Hz}$} & 4WICS & 0.0563 & 0.0436 & 0.0859 & 0.0224 \\
\hline & Traditional & 0.0464 & 0.0385 & 0.0246 & 0.0360 \\
\hline & Comparison \% & $21.34 \uparrow$ & $13.25 \uparrow$ & $249.2 \uparrow$ & $37.78 \downarrow$ \\
\hline \multirow{3}{*}{$6 \mathrm{~Hz}$} & 4WICS & 0.0263 & 0.2946 & 0.0015 & 0.0024 \\
\hline & Traditional & 0.0348 & 0.0407 & 0.0102 & 0.0199 \\
\hline & Comparison \% & $24.43 \downarrow$ & $623.8 \uparrow$ & $85.29 \downarrow$ & $87.94 \downarrow$ \\
\hline \multirow{3}{*}{$7 \mathrm{~Hz}$} & 4WICS & 0.0349 & 0.0757 & 0.0142 & 0.0559 \\
\hline & Traditional & 0.0075 & 0.0349 & 0.0015 & 0.0043 \\
\hline & Comparison \% & $365.3 \uparrow$ & $116.9 \uparrow$ & $846.7 \uparrow$ & $1200 \uparrow$ \\
\hline \multirow{3}{*}{$8 \mathrm{~Hz}$} & 4WICS & 0.0506 & 0.0179 & 0.0391 & 0.0078 \\
\hline & Traditional & 0.0121 & 0.0182 & 0.0093 & 0.0068 \\
\hline & Comparison \% & $318.2 \uparrow$ & $1.648 \downarrow$ & $320.4 \uparrow$ & $14.71 \uparrow$ \\
\hline \multirow{3}{*}{$9 \mathrm{~Hz}$} & 4WICS & 0.0201 & 0.0023 & 0.0192 & 0.0054 \\
\hline & Traditional & 0.0205 & 0.0052 & 0.0014 & 0.0166 \\
\hline & Comparison \% & $1.951 \downarrow$ & $55.77 \downarrow$ & $1271 \uparrow$ & $67.47 \downarrow$ \\
\hline \multirow{3}{*}{$10 \mathrm{~Hz}$} & $4 \mathrm{WICS}$ & 0.0023 & 0.0076 & 0.0031 & 0.0037 \\
\hline & Traditional & 0.0276 & 0.0415 & 0.0041 & 0.0127 \\
\hline & Comparison \% & $91.67 \downarrow$ & $81.69 \downarrow$ & $24.39 \downarrow$ & $70.87 \downarrow$ \\
\hline
\end{tabular}

\section{Conclusion}

The configuration of a 5-DOF vehicle model with 4WICS is introduced for space force analysis. On the basis of the described dynamical model, which includes a center layer and four controllable local layers, a hierarchical modeling control method is developed. The computational process for the hierarchical modeling control is also addressed. Numerical simulations and comparisons between the 4WICS control and traditional active vibration control are carried out under the conditions of bump and C-grade road excitations. Owing to the use of parallel computation for local layers in the hierarchical modeling control method, the calculation time for the suspension performance is shortened and the system response also becomes rapid, thereby realizing detailed and precise control in response to external excitations. Moreover, the vehicle ride comfort and driving stability are also improved. 


\section{Acknowledgments}

The authors acknowledge the financial support from the Training Program of Outstanding Discipline and Specialty Leaders of Fujian Province Universities and Institutions, the China postdoctoral fund (2013M541851), and the Operational Funding of the Advanced Talents for Scientific Research (19YG04) supported by Sanming University. Technical supports from the following institutions are also acknowledged: Fujian Public Service Platform for miniEREV Power System, Fujian Provincial University Engineering Research Center for Modern Mechanical Design and Manufacturing Technology, Fujian Provincial Collaborative Innovation Center for Green Casting, Forging and Advanced Manufacturing, and Fujian Provincial Engineering Research Center for Casting and Forging Parts.

\section{References}

1 Z.Q.M. Chen, Y. Hu, and F. Wang: J. Dyn. Syst. Meas. Control. 137 (2015) 1. https://doi.org/10.1115/1.4031630

2 Y. Huang, J. Na, X. Wu, X. Liu, and Y. Guo: ISA Trans. 54 (2015) 145. https://doi.org/10.1016/j.isatra.2014.05.025

3 Y. Huang, J. Na, X. Wu, G.B. Gao, and Y. Guo: Trans. Inst. Meas. Control. 40 (2018) 1237. https://doi. org/10.1177/0142331216678312

4 J. Na, Y. Huang, X. Wu, G.B. Gao, G. Herrmann, and J. Z. Jiang: IEEE Trans. Control Syst. Tech. 26 (2018) 2063. https://doi.org/2018. 10.1109/TCST.2017.2746060

5 H. Pan, W.C Sun, and X. Jing: J. Sound Vib. 399 (2017) 2. https://doi.org/10.1016/j.jsv.2017.03.011

6 R. Wang, H. Jing, and K.H. Reza: Mech. Syst. Signal Proc. 62/63 (2015) 341. https://doi.org/10.1016/ j.ymssp.2015.01.015

7 M. Morteza and F. Afef: IEEE Trans. Control Syst. Tech. 23 (2015) 1164. https://doi.org/10.1109/ TCST.2014.2357341

8 H. Zhang, R. Wang, J. Wang, and Y. Shi: Mechatronics 24 (2014) 354. https://doi.org/10.1016/ j.mechatronics.2013.07.013

9 H. Hui, R. Wang, C. Li, J. Wang, and N. Chen: Int. J. Veh. Design. 68 (2015) 22. https://doi.org/10.1504/ IJVD.2015.071067

10 Y. Guan, Q.L. Han, H. Yao, and X. Ge: Nonlinear Dyn. 94 (2018) 627. https://doi.org/10.1007/s11071-018-43810

11 G. Wang, M. Chadli, H. Chen, and Z. Zhou: J. Franklin Inst. 356 (2019) 147. https://doi.org/10.1016/ j.jfranklin.2018.10.012

12 Z.J. Fu, B. Li, X.B. Ning, and W.D. Xie: Math. Prob. Eng. 2017 (2017) 4575926. https://doi. org/10.1155/2017/4575926

13 X. Shao, N. Fazel, and H. Du: Mech. Syst. Signal Proc. 87 (2017) 365. https://doi.org/10.1016/j.ymssp.2016.10.032

14 H. Li, X. Jing, L.H. Keung, and P. Shi: IEEE Trans. Cyber. 44 (2014) 1111. https://doi.org/10.1109/ TCYB.2013.2279534

15 G.I.Y Mustafa, H.P. Wang, and T. Yang: Adv. Eng. Soft. 127 (2019) 141. https://doi.org/10.1016/ j.advengsoft.2018.04.009

16 A.A. Aldair and W.J. Wang: J. Vib. Control. 18 (2011) 1837. https://doi.org/10.1177/1077546311428631

17 L. Khan, S. Qamar, and M.U. Khan: Arabian J. Sci. Eng. 39 (2014) 2045. https://doi.org/10.1007/s13369-0130729-4

18 S.D. Nguyen, Q.H. Nguyen, and S.B. Choi: Mech. Syst. Signal Proc. 56/57 (2015) 288. https://doi.org/10.1016/ j.ymssp.2014.10.019

19 H. Pang, X. Zhang, and Z. Xu: ISA Trans. 88 (2019) 23. https://doi.org/10.1016/j.isatra.2018.11.047

20 H. Pang, X. Zhang, J. Chenand, and K. Liu: App. Math. Mode. 76 (2019) 479. https://doi.org/10.1016/ j.apm.2019.06.030

21 G. Wang: Proc. Inst. Mech. Eng. Part I, J. Syst. Control Eng. 231 (2017) 459. https://doi. org/10.1177/0959651817704537

22 U. S. Pusadkar, S. D. Chaudhari, P. D. Shendge, and S. B. Phadke: J. Sound Vib. 442 (2019) 428. https://doi. org/10.1016/j.jsv.2018.11.003

23 H. P. Wang, G. I. Y. Mustafa, and Y. Tian: Adv. Eng. Soft. 115 (2018) 452. https://doi.org/10.1016/ j.advengsoft.2017.11.001 
24 H. Gao, W. Sun, and P. Shi: IEEE Trans. Control Syst. Tech. 18 (2010) 238. https://doi.org/10.1109/ TCST.2009.2015653.

25 Y. Kyongsu and H. Karl: J. Dyn. Syst. Meas. Control: 117 (1995). https://doi.org/10.1115/1.2835177.

26 J. Marzbanrad, G. Ahmadi, H. Zohoor, and Y. Hojjat: J. Sound Vib. 275 (2004) 973. https://doi.org/10.1016/ s0022-460x(03)00812-5.

27 P. Gáspár, Z. Szabo, and J. Bokor: Int. J. Veh. Syst. Model. Testing 2 (2007). https://doi.org/10.1504/ ijvsmt.2007.013891.

28 I. Youn, M. A. Khan, N. Uddin, E. Youn, and M. Tomizuka: Int. J. Auto. Tech. 18 (2017) 307. https://doi. org/10.1007/s12239.017.0031.7.

29 H. Du, W. Li, and N. Zhang: Int. J. Veh. Design. 63 (2013) 159. https://doi.org/10.1504/ijvd.2013.056133

30 B. Mohamed and J.R. Marc: Eur. J. Mech. A/Solids 20 (2001) 509. https://doi.org/10.1016/S09977538(01)01138-X

\section{Nomenclature}

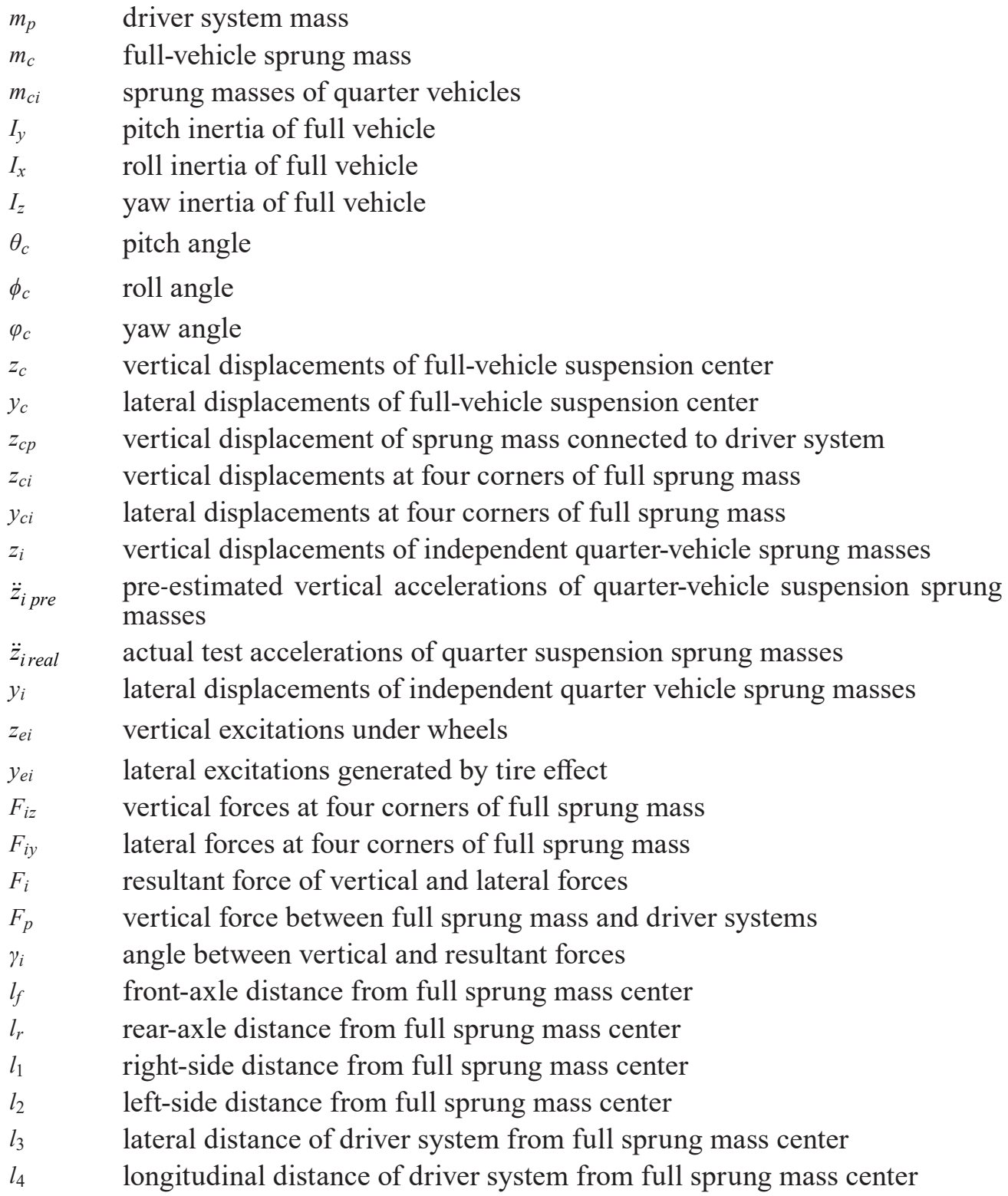




\section{Appendix}

$\lambda=\sin \gamma_{1} \sin \gamma_{3} \cos \gamma_{2} \cos \gamma_{4} l\left(l_{3}-l_{2}\right)+\sin \gamma_{1} \sin \gamma_{4} \cos \gamma_{2} \cos \gamma_{3}\left(l_{2}\left(l_{r}+l_{4}\right)+l_{1}\left(l_{4}-l_{f}\right)-l l_{3}\right)$

$+\sin \gamma_{2} \sin \gamma_{3} \cos \gamma_{1} \cos \gamma_{4}\left(l_{2}\left(l_{f}-l_{4}\right)-l_{1}\left(l_{r}+l_{4}\right)-l l_{3}\right)+\sin \gamma_{2} \sin \gamma_{4} \cos \gamma_{1} \cos \gamma_{3} l\left(l_{1}+l_{3}\right)$

$\lambda_{11}=\left[\sin \gamma_{3} \cos \gamma_{4}\left(l_{r} l_{3}+l_{2} l_{4}\right)+\sin \gamma_{4} \cos \gamma_{3}\left(l_{1} l_{4}-l_{r} l_{3}\right)\right] \sin \gamma_{2}$

$\lambda_{12}=\sin \gamma_{3} \cos \gamma_{2} \cos \gamma_{4} l_{r}\left(l_{2}-l_{3}\right)+\sin \gamma_{4} \cos \gamma_{2} \cos \gamma_{3}\left[l l_{3}-l_{2}\left(l_{r}+l_{4}\right)+l_{1}\left(l_{f}-l_{4}\right)\right] l_{r} / l-\sin \gamma_{2} \cos \gamma_{3} \cos \gamma_{4} l_{f}\left(l_{1}+l_{2}\right)\left(l_{r}+l_{4}\right) / l$

$\lambda_{13}=\sin \gamma_{2} \sin \gamma_{3} \cos \gamma_{4}\left(l_{2}-l_{3}\right)+\sin \gamma_{2} \sin \gamma_{4} \cos \gamma_{3}\left(l_{1}+l_{3}\right)$

$\lambda_{14}=\left(\sin \gamma_{3} \cos \gamma_{4}-\sin \gamma_{4} \cos \gamma_{3}\right) \sin \gamma_{2}\left(l_{r}+l_{4}\right)$

$\lambda_{15}=\sin \gamma_{2} \cos \gamma_{3} \cos \gamma_{4}\left(l_{1}+l_{2}\right)\left(l_{r}+l_{4}\right) / l+\sin \gamma_{3} \cos \gamma_{2} \cos \gamma_{4}\left(l_{2}-l_{3}\right)+\sin \gamma_{4} \cos \gamma_{2} \cos \gamma_{3}\left(l_{1}\left(l_{f}-l_{4}\right)-l_{2}\left(l_{r}+l_{4}\right) / l+l_{3}\right)$

$\lambda_{21}=\left[\sin \gamma_{1} \sin \gamma_{3} \cos \gamma_{4}\left(l_{2} l_{4}+l_{r} l_{3}\right)+\sin \gamma_{1} \sin \gamma_{4} \cos \gamma_{3}\left(l_{1} l_{4}-l_{r} l_{3}\right)\right]$

$\lambda_{22}=\sin \gamma_{3} \cos \gamma_{1} \cos \gamma_{4}\left(l_{2}\left(l_{f}-l_{4}\right)-l l_{3}-l_{1}\left(l_{r}+l_{4}\right)\right) l_{r} / l+\sin \gamma_{4} \cos \gamma_{1} \cos \gamma_{3} l_{r}\left(l_{1}+l_{3}\right)-\sin \gamma_{1} \cos \gamma_{3} \cos \gamma_{4}\left(l_{1}+l_{2}\right)\left(l_{r}+l_{4}\right) l_{f} / l$

$\lambda_{23}=\left[\sin \gamma_{3} \cos \gamma_{4}\left(l_{2}-l_{3}\right)+\sin \gamma_{4} \cos \gamma_{3}\left(l_{1}+l_{3}\right)\right] \sin \gamma_{1}$

$\lambda_{24}=\left(\sin \gamma_{3} \cos \gamma_{4}-\sin \gamma_{4} \cos \gamma_{3}\right) \sin \gamma_{1}\left(l_{r}+l_{4}\right)$

$\lambda_{25}=\sin \gamma_{1} \cos \gamma_{3} \cos \gamma_{4}\left(l_{1}+l_{2}\right)\left(l_{r}+l_{4}\right) / l+\sin \gamma_{4} \cos \gamma_{1} \cos \gamma_{3}\left(l_{1}+l_{3}\right)+\sin \gamma_{3} \cos \gamma_{1} \cos \gamma_{4}\left[l_{2}\left(l_{f}-l_{4}\right)-l_{1}\left(l_{r}+l_{4}\right) / l-l_{3}\right]$

$\lambda_{31}=\sin \gamma_{1} \sin \gamma_{4} \cos \gamma_{2}\left(l_{2} l_{4}-l_{f} l_{3}\right)+\sin \gamma_{2} \sin \gamma_{4} \cos \gamma_{1}\left(l_{f} l_{3}+l_{1} l_{4}\right)$

$\lambda_{32}=\sin \gamma_{1} \cos \gamma_{2} \cos \gamma_{4} l_{f}\left(l_{3}-l_{2}\right)+\sin \gamma_{2} \cos \gamma_{1} \cos \gamma_{4}\left(l_{2}\left(l_{f}-l_{4}\right)-l_{1}\left(l_{r}+l_{4}\right)-l l_{3}\right) l_{f} / l+\sin \gamma_{4} \cos \gamma_{1} \cos \gamma_{2}\left(l_{f}-l_{4}\right)\left(l_{1}+l_{2}\right) / l$

$\lambda_{33}=\sin \gamma_{1} \sin \gamma_{4} \cos \gamma_{2}\left(l_{2}-l_{3}\right)+\sin \gamma_{2} \sin \gamma_{4} \cos \gamma_{1}\left(l_{1}+l_{3}\right)$

$\lambda_{34}=\left(\sin \gamma_{2} \cos \gamma_{1}-\sin \gamma_{1} \cos \gamma_{2}\right) \sin \gamma_{4}\left(l_{f}-l_{4}\right)$

$\lambda_{35}=\sin \gamma_{1} \cos \gamma_{2} \cos \gamma_{4}\left(l_{2}-l_{3}\right)+\sin \gamma_{2} \cos \gamma_{1} \cos \gamma_{4}\left[l_{3}+l_{2}\left(l_{4}-l_{f}\right)+l_{1}\left(l_{r}+l_{4}\right) / l\right]+\sin \gamma_{4} \cos \gamma_{1} \cos \gamma_{2}\left[\left(l_{1}+l_{2}\right)\left(l_{f}-l_{4}\right)\right] / l$

$\lambda_{41}=\sin \left(\gamma_{e 1}\right) \sin \left(\gamma_{e 3}\right) \cos \left(\gamma_{e 2}\right) l_{f} l_{3}-\sin \left(\gamma_{e 2}\right) \sin \left(\gamma_{e 3}\right) \cos \left(\gamma_{e 1}\right) l_{1} l_{4}-\sin \left(\gamma_{e 2}\right) \sin \left(\gamma_{e 3}\right) \cos \left(\gamma_{e 1}\right) l_{f} l_{3}-\sin \left(\gamma_{e 1}\right) \sin \left(\gamma_{e 3}\right) \cos \left(\gamma_{e 2}\right) l_{2} l_{4}$

$\lambda_{42}=\sin \gamma_{1} \cos \gamma_{2} \cos \gamma_{3}\left\{\left[l_{f} l_{2}\left(l_{r}+l_{4}\right)+l_{f} l_{1}\left(l_{4}-l_{f}\right)\right] / l-l_{f} l_{3}\right\}+\sin \gamma_{2} \cos \gamma_{1} \cos \gamma_{3} l_{f}\left(l_{1}+l_{3}\right)+\sin \gamma_{3} \cos \gamma_{1} \cos \gamma_{2}\left(l_{4}-l_{f}\right)\left(l_{1}+l_{2}\right) l_{r} / l$

$\lambda_{43}=\sin \gamma_{3}\left[\sin \gamma_{1} \cos \gamma_{2}\left(l_{3}-l_{2}\right)-\sin \gamma_{2} \cos \gamma_{1}\left(\left(l_{f}-l_{r}\right) l_{1} / l-l_{3}\right)\right]$

$\lambda_{44}=\left(\sin \gamma_{1} \cos \gamma_{2}-\sin \gamma_{2} \cos \gamma_{1}\right) \sin \gamma_{3}\left(l_{f}-l_{4}\right)$

$\lambda_{45}=\sin \gamma_{1} \cos \gamma_{2} \cos \gamma_{3}\left(l_{3}+\frac{l_{1}\left(l_{f}-l_{4}\right)-l_{2}\left(l_{r}+l_{4}\right)}{l}\right)-\sin \gamma_{2} \cos \gamma_{1} \cos \gamma_{3}\left(l_{1}+l_{3}\right)+\sin \gamma_{3} \cos \gamma_{1} \cos \gamma_{2} \frac{\left(l_{1}+l_{2}\right)\left(l_{4}-l_{f}\right)}{l}$

$\lambda_{51}=\sin \gamma_{1} \sin \gamma_{3} \cos \gamma_{2} \cos \gamma_{4} l l_{2}+\sin \gamma_{2} \sin \gamma_{3} \cos \gamma_{1} \cos \gamma_{4}\left(l_{r} l_{1}-l_{f} l_{2}\right)$

$+\sin \gamma_{1} \sin \gamma_{4} \cos \gamma_{2} \cos \gamma_{3}\left(l_{f} l_{1}-l_{r} l_{2}\right)+\sin \gamma_{2} \sin \gamma_{4} \cos \gamma_{1} \cos \gamma_{3}\left(l_{r}-l_{f}\right) l_{1}$

$\lambda_{52}=\left[\left(\sin \gamma_{2} \cos \gamma_{4}+\sin \gamma_{4} \cos \gamma_{2}\right) \cos \gamma_{1} \cos \gamma_{3}-\left(\sin \gamma_{1} \cos \gamma_{3}+\sin \gamma_{3} \cos \gamma_{1}\right) \cos \gamma_{2} \cos \gamma_{4}\right] l\left(l_{1}+l_{2}\right)$

$\lambda_{53}=\left(\sin \gamma_{1} \sin \gamma_{4} \cos \gamma_{2} \cos \gamma_{3} l_{2}-\sin \gamma_{2} \sin \gamma_{3} \cos \gamma_{1} \cos \gamma_{4} l_{2}\right)\left(l_{1}+l_{2}\right)$

$\lambda_{54}=\left[\sin \gamma_{1} \sin \gamma_{3} \cos \gamma_{2} \cos \gamma_{4}-\sin \gamma_{2} \sin \gamma_{3} \cos \gamma_{1} \cos \gamma_{4}+\sin \gamma_{2} \sin \gamma_{4} \cos \gamma_{1} \cos \gamma_{3}-\sin \gamma_{1} \sin \gamma_{4} \cos \gamma_{2} \cos \gamma_{3}\right] l$

$\lambda_{55}=\left[\sin \gamma_{1} \cos \gamma_{2} \cos \gamma_{3} \cos \gamma_{4}-\sin \gamma_{2} \cos \gamma_{1} \cos \gamma_{3} \cos \gamma_{4}+\sin \gamma_{4} \cos \gamma_{1} \cos \gamma_{2} \cos \gamma_{3}-\sin \gamma_{3} \cos \gamma_{1} \cos \gamma_{2} \cos \gamma_{4}\right]\left(l_{1}+l_{2}\right)$ 